\title{
Maternal genital chlamydial infection as a cause of neonatal conjunctivitis
}

\author{
D. HOBSON \\ M.D., M.R.C.Path. \\ ElisABETH REES \\ M.D. \\ Departments of Medical Microbiology and Venereology, University of Liverpool, Liverpool
}

\begin{abstract}
Summary
Infections of the cervix with Chlamydia trachomatis are common, at least in those groups of sexually active women of child-bearing age who are seen in STD (sexually-transmitted diseases) clinics. Persistent untreated infection presents a hazard to the women themselves during pregnancy and to their infants who may develop chlamydial conjunctivitis.

The clinical and laboratory findings in 1009 women and in 103 infants with conjunctivitis are presented. Practical problems of diagnosis and control of neonatal chlamydial conjunctivitis are described. A possible association between prematurity and chlamydial infection is discussed.
\end{abstract}

\section{Introduction}

The control of many types of perinatal infection must begin with the recognition of the pathogenic agent in pregnant women and its eradication, whereever possible, before their babies are born. Chlamydial infections clearly illustrate this, and show how quickly a perinatal infective syndrome may arise, apparently de novo, in communities where an infection of young women has become common, but has escaped general recognition and treatment. This review presents some results of a combined clinical and laboratory survey of infection with Chlamydia trachomatis (TRIC* agents) in Liverpool from September 1973 to September 1976.

\section{Material and methods}

Method of selection and examination of patients have been described elsewhere (Rees et al., 1977a, b, c).

Laboratory procedures for isolating $C$. trachomatis $(\mathrm{Ct})$ in replicating cultures of $\mathrm{McCoy}$ cells were those described by Hobson et al. (1974) and Johnson and Hobson (1976).

Correspondence: Dr D Hobson, Department of Medical Microbiology, New Medical School, University of Liverpool (P.O. Box 147), Liverpool L69 3BX.

\footnotetext{
* Trachoma-inclusion conjunctivitis.
}

Results

Cervical swabs were Ct-positive in $282(28 \%)$ of 1009 women attending a single sexually transmitted diseases (STD) clinic. Women who were known at the time of their first visit to have gonorrhoea $(G)$, or to be contacts of men with $G$ were not included in this series.

Ct-positive cervical swabs were found in seventyone $(35 \%)$ of 203 consecutive patients who were the reported female sexual contacts of men with nongonococcal urethritis.

Swabs from the eyes of 103 newborn infants with conjunctivitis were $\mathrm{Ct}$-positive in thirty-three $(33 \%)$ cases. In one of these Ct-positive infants herpes simplex virus was also isolated, in three others Neisseria gonorrhoeae was found and Staphylococcus pyogenes in two cases. In eight of the sixty-nine Ct-negative infants, $\mathrm{G}$ was isolated, and Staph. pyogenes, streptococci or coliform organisms were found in a further eight infants.

\section{Discussion and conclusions}

Women who choose to visit, or are referred to, an STD clinic are not likely to be representative of the general female population, and the high incidence of $\mathrm{Ct}$ infection found here may reflect a certain lifestyle and a considerable degree of self-selection. However, all these patients were sexually active women of child-bearing age (range 14-37 years) and all social classes were represented. Many were asymptomatic, with no prior reason to suspect infection since they were the non-promiscuous wives of men with extramarital sexual contacts. In a large city, such young women may be a significant proportion of all those who add regularly to the neonatal population. Chlamydial infection was frequently associated with cervical lesions which could persist for several months in the absence of correct chemotherapy (Rees et al., 1977b).

The possibility of a continuing reservoir of chlamydial infection in potential mothers prompted an investigation of newborn infants with 'sticky eye'. 
The group of infants reported here is not representative of 'sticky eye' in general and excludes many trivial examples; this is a common condition in maternity units, and can result from infection by a number of different microbial agents (Csonka and Coufalik, 1977) or even from trauma during birth. However, the main element of deliberate selection in the present series was that most of the infants were referred to the authors, by the doctor in charge of their primary care, because they had an active conjunctivitis which had failed to respond to neomycin (or less commonly chloramphenicol) eye drops, or for which no bacterial or viral cause had been found. Details of the history, clinical features and laboratory findings of these infants have been discussed by Rees et al. (1977a).

For the specific purpose of this symposium, only the practical problems that can arise in the detection and control of this particular example of perinatal infection will be discussed.

The age of the infant when the discharge began ranged from 3-13 days (mean, 6.7 days). Hence, many infants could be sent home from the maternity unit during the incubation period, with a risk that the subsequent conjunctivitis would not be diagnosed early or accurately and that the correct treatment would be delayed.

Untreated chlamydial conjunctivitis often progressed rapidly (Fig. 1) with gross oedema of the eyelids, congestion and 'bubbling' of the conjunctiva and muco-purulent discharge from which $\mathrm{Ct}$ could be isolated in large numbers. In some cases, however, the clinical signs were less dramatic, particularly when chloramphenicol eye drops or systemic penicillins had been given at an early stage. These antibiotics could mndify the clinical picture and reduce the number of $\mathrm{Ct}$ particles capable of growing in tissue culture, but often failed to eradicate the $\frac{\varrho}{3}$ infection, with recrudescence when the antibiotic was $\stackrel{\curvearrowright}{\propto}$ discontinued (Rees et al., 1977a). When oxytetra- $c$. cycline eye ointment was given daily for 1 month, eighteen of the twenty-four infants followed-up $\stackrel{5}{+}$ became Ct-negative; the signs of conjunctivitis disappeared quickly and did not recur. Ct was re- $\frac{\bar{O}}{\bar{D}}$ isolated from six infants after treatment and, in five $\frac{\rho}{\sigma}$

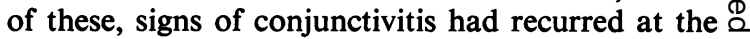
time of re-isolation. In two of the relapses the original course of tetracycline had been cut short or $\vec{\circ}$ misapplied.

Active disease persisted in infants whose $\mathrm{Ct}$ in- $\overrightarrow{\vec{\omega}}$ fection was not detected before they left hospital and $\stackrel{\rho}{\circ}$ who had not received tetracyclines; $\mathrm{Ct}$ was isolated 00 from seven infants in this category at $13,17,19,3$. 24 (two), 30 or 57 days of age respectively. Such $\mathcal{E}$ continuing infection could involve risks both to the infant and to his family. Firstly, permanent eye $\stackrel{N}{+}$ damage such as that seen in tropical trachoma could of possibly occur, especially in unhygienic homes where $\mathcal{G}$ bacterial superinfection of the eye is likely. Micro- $\frac{}{工}$ pannus and conjunctival scarring in the eyes of $\vec{O}$ children with $\mathrm{Ct}$ infection has been reported by Mordhorst and Dawson (1971). Secondly, Ct may $\frac{0}{\circ}$ spread from the infant to other members of the $\stackrel{\Phi}{-}$ family, as is frequent in tropical trachoma (Jone $\overrightarrow{0}$ 1975). In the present series, $\mathrm{Ct}$ was isolated from 18-month-old sibling of an infant whose conjuncti vitis had developed 6 days after birth but was no diagnosed until the eighteenth day. This toddler's conjunctivitis began 4 days after the infant had been taken back into hospital.

An unexpected finding was that fourteen of thirtythree $(42 \%)$ of the Ct-positive infants were premature, $\overrightarrow{\vec{O}}$ compared with only nine of fifty-eight $(15.8 \%)$ of 3 Ct-negative infants. It seems possible that maternal

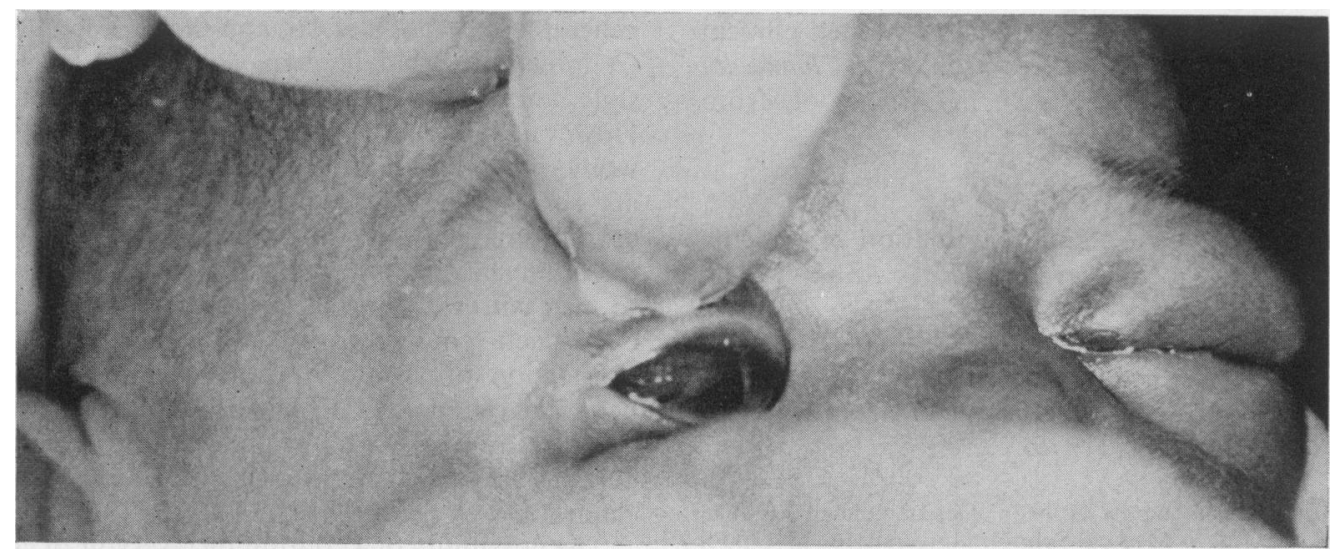

FIG. 1. Bilateral conjunctivitis in a 6-day-old infant, showing gross oedema of the eyelids and mucosa. Chlamydia trachomatis was isolated from both eyes and from the mother's cervix (shown in Fig. 2). 


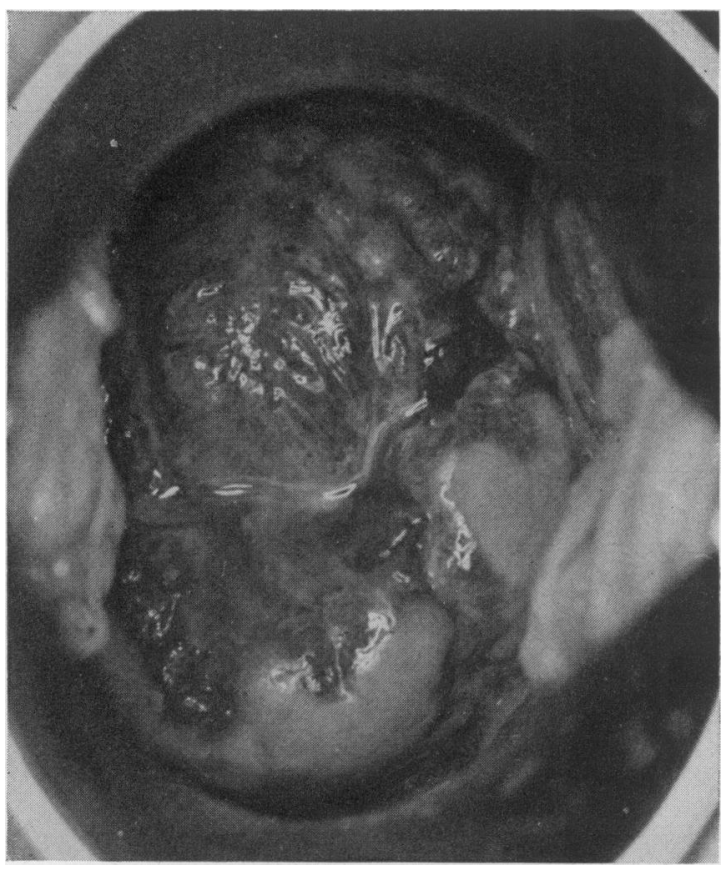

FIG. 2. The appearance of the cervix in the mother of the baby with neonatal conjunctivitis (shown in Fig. 1). Chlamydia trachomatis was isolated from both mother and infant. The cervix shows a large hypertrophic erosion, and muco-purulent discharge at the os.

Ct infection might have been responsible for both the prematurity and the conjunctivitis. Many infected pregnant women had large cervical erosions and endocervicitis (Fig. 2). The finding that nineteen of thirty-one mothers of Ct-positive infants developed post-partum infection shortly after delivery suggests that these lesions might give rise to more generalized infections (Rees et al., 1977c). It seems probable that $\mathrm{Ct}$ infection may already be extending late in pregnancy to involve the amnion overlying the cervical canal, thus weakening the membranes sufficiently to cause premature rupture and early labour and per- haps infecting the infant's eyes in utero in some cases. It may be significant that one infant with $\mathrm{Ct}$ positive conjunctivitis in this series was born by Caesarian section; on further investigation it seemed unlikely that post-natal spread from the infected mother to the infant could have occurred. More detailed studies to explore the relationship between prematurity and chlamydial infection are in progress to determine whether conjunctivitis is only one of the hazards to which the offspring of a woman with chlamydial genital infection is exposed.

\section{Acknowledgments}

The authors are greatly indebted to the Medical Research Council for a project grant to support these studies, and to our colleagues Dr I. Anne Tait, Mr F. W. A. Johnson and Mrs Ruth E. Byng for their contribution to the findings presented here.

\section{References}

Csonka, G.W. \& Coufalik, E.D. (1977) Chlamydial, gonococcal, and herpes virus infections in neonates. Postgraduate Medical Journal, 53, 592.

Hobson, D., Johnson, F.W.A., Rees, E. \& TAIT, I.A. (1974 Simplified method for diagnosis of genital and ocular infections with chlamydia. Lancet, ii, 555.

JoHnSON, F.W.A. \& HoBSON, D. (1976) Factors affecting the sensitivity of replicating McCoy cells in the isolation and growth of chlamydia A (TRIC agents). Journal of Hygiene, 76, 441.

JoNES, B.R. (1975) The prevention of blindness from trachoma. Transactions of the Ophthalmological Societies of the United Kingdom, 95, 16.

Mordhorst, C.H. \& Dawson, C. (1971) Sequelae of neonatal inclusion conjunctivitis and associated disease in parents. American Journal of Ophthalmology, 71, 861.

Rees, E., Tait, I.A., Hobson, D., Byng, R.E. \& Johnson, F.W.A. (1977a) Neonatal conjunctivitis caused by Neisseria gonorrhoeae and Chlamydia trachomatis. British Journal of Venereal Diseases, 51, 173.

ReEs, E., TAIT, I.A., Hobson, D. \& Johnson, F.W.A. (1977b) Chlamydia in relation to cervical infection and pelvic inflammatory disease. In: Non-Gonococcal Urethritis and Related Infections. (Ed. by D. Hobson and K. K. Holmes). American Society for Microbiology.

ReEs, E., TAIT, I.A., Hobson, D. \& Johnson, F.W.A. (1977c) Perinatal chlamydial infections. In: Non-gonococcal Urethritis and Related Infections (Ed. by D. Hobson and K. K. Holmes). 